\title{
SCHEMES OFFER GRANTS TO STIMULATE RESEARCH
}

The Academy of Medical Sciences has launched a $£ 1$ million scheme called 'Inspire' to stimulate medical and dental undergraduates to pursue scientific research, with funding from the Wellcome Trust. The scheme aims to foster a research culture in all clinicians entering the NHS to ensure patients benefit from the latest breakthroughs in medical science.

Inspire is a five year, UK-wide scheme that will provide grants of up to $£ 10,000$ to medical schools to establish innovative activities that build student interest in research. A special project fund of $£ 100,000$ will also be distributed to support particularly innovative or high impact activities. The Academy will run a series of sharing conferences to enable medical schools to showcase activities and share learning.

The Academy's Starter Grants for Clinical Lecturers scheme offers $£ 4.5$ million in start-up funds to help doctors and dentists kick start their scientific career by allowing them to pursue academic work alongside patient care.

The money will be distributed in grants of up to $£ 30,000$ to clinical lecturers to help them gather preliminary data and strengthen their applications for longer term fellowships and funding. The grants will be awarded biannually from 2013 for four years through the Academy of Medical Sciences Starter Grants for Clinical Lecturers Scheme.

Further details for both schemes are available at www.acmedsci.ac.uk.

\section{DIFFICULTY CHEWING LINKED TO DEMENTIA RISK}

A Swedish study ${ }^{1}$ has established an association between chewing ability and the risk of dementia, with tooth loss and difficulty chewing hard foods increasing the odds of cognitive impairment.

A nationwide sample of 557 people aged 77 or older participated in the study measuring cognitive and masticatory function. Dental status and chewing ability was acquired through self-assessment and it was discovered that whilst the level of risk of cognitive impairment was not significantly different between participants with natural teeth or those with multiple tooth loss and dentures, the odds of impairment were significantly higher for those experiencing chewing difficulties. Even when taking into account gender, age, education and mental illness, the positive correlation between difficulty chewing and increased dementia risk remained unchanged.

One possible explanation behind these results is that less chewing leads to less blood flow to the brain, though further research is needed. In our ageing population, it is estimated that 820,000 people in the UK suffer from dementia, with 4.6 million new cases diagnosed per year worldwide. The evidence collected from this study could prove significant in the ongoing research into the aetiology of dementia and potential methods for slowing its progression.

1. Lexomboon D, Trulsson M, Wårdh I, Parker M G. Chewing ability and tooth loss: associated with cognitive impairment in an elderly population study. J Am Geriatr Soc 2012; epub ahead of print.

2. Alzheimer's Research UK. Dementia statistics. Cambridge: Alzheimer's Research UK, 2012.

By Laura Pacey

\section{FELLOWSHIP WITHOUT EXAMINATION LAUNCHED}

The Royal College of Surgeons of Edinburgh's Faculty of Dental Surgery has launched a new Dental Fellowship without examination.

This new route to Fellowship allows dental professionals holding a Specialty Dental Membership an opportunity to access the FDS RCS(Edin) without examination through written application. It is a qualification of the highest order which previously few had the opportunity to achieve, and one which no other Royal Surgical College currently offers. 